\title{
Small-Molecule Inhibition of UBE2T/FANCL-Mediated Ubiquitylation in the Fanconi Anemia Pathway
}

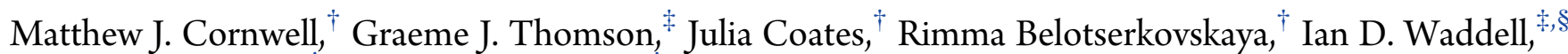 \\ Stephen P. Jackson, ${ }^{\dagger}$ and Yaron Galanty*, ${ }^{*}{ }^{\dagger}$ \\ ${ }^{\dagger}$ The Wellcome Trust/Cancer Research UK Gurdon Institute and Department of Biochemistry, University of Cambridge, \\ Cambridge CB2 1QN, United Kingdom \\ ${ }^{\ddagger}$ Drug Discovery Unit, Cancer Research UK Manchester Institute, University of Manchester, Wilmslow Road, Manchester M20 \\ 4BX, United Kingdom
}

\section{Supporting Information}

ABSTRACT: The Fanconi anemia pathway orchestrates the repair of DNA interstrand cross-links and stalled replication forks. A key step in this pathway is UBE2T and FANCLdependent monoubiquitylation of the FANCD2-FANCI complex. The Fanconi anemia pathway represents an attractive therapeutic target, because activation of this pathway has been linked to chemotherapy resistance in several cancers. However, to date, very few selective inhibitors of ubiquitin conjugation pathways are known. By using a high-throughput screencompatible assay, we have identified a small-molecule inhibitor

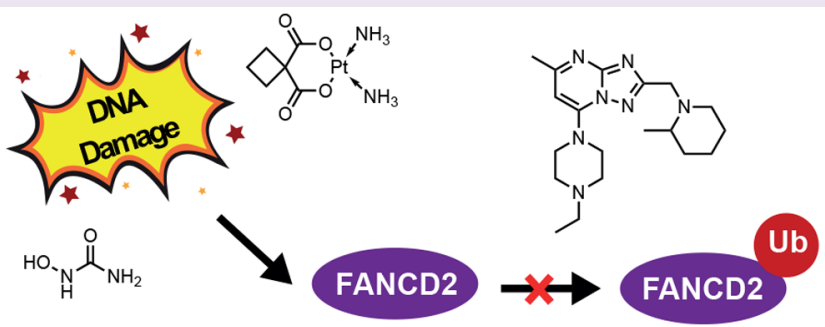
of UBE2T/FANCL-mediated FANCD2 monoubiquitylation that sensitizes cells to the DNA cross-linking agent, carboplatin.

U biquitylation is a post-translational modification that regulates a myriad of cellular processes. Proteins are ubiquitylated via an enzymatic cascade composed of E1 activating, E2 conjugating, and E3 ligating enzymes. ${ }^{1}$ Because of the crucial physiological role of the ubiquitin system, its dysregulation is implicated in a growing number of human pathologies, including several cancers, developmental defects, immunodeficiencies, and neurodegenerative disorders. ${ }^{2}$ Ubiquitylation is known to play key roles in a vast array of proteolytic and nonproteolytic regulatory mechanisms. One area in particular where ubiquitylation events are highly prevalent is in the DNA damage response (DDR). ${ }^{3}$

Genome integrity is continuously under attack from a barrage of exogenous and endogenous genotoxic agents such as ionizing radiation, ultraviolet light (UV) radiation and oxidative stress, and by errors in DNA replication itself. Fortunately, cells possess highly efficacious mechanismscollectively known as the DDR-which are able to, among other things, detect DNA lesions, activate cell cycle checkpoints, and repair the damaged DNA. ${ }^{4}$

The Fanconi anemia (FA) pathway, also known as the FA/ BRCA pathway, is required for the repair of DNA interstrand cross-links (ICLs). ${ }^{5}$ ICLs are among the most cytotoxic forms of DNA lesion, and occur when bases from opposite DNA strands become covalently attached to each other. ICLs inhibit essential processes such as replication and transcription and must be repaired or bypassed for the cell to survive.

ICL-inducing anticancer agents, such as platinum-based compounds (including cisplatin and carboplatin) and mitomycin $\mathrm{C}$, have long been used in the clinic to treat a range of malignancies including testicular, ovarian, head and neck, colorectal, bladder, and lung cancers. ${ }^{6}$ Although these chemotherapies are generally initially effective at cytoreduction, tumor recurrence and drug resistance commonly arise. Activation or upregulation of the FA pathway has been linked to chemotherapy resistance in several cancers; therefore, its inhibition is hypothesized to restore sensitivity to ICLinducing agents.

Currently, 22 genes are annotated as FA genes (FANCA to FANCW; http://www2.rockefeller.edu/fanconi/mutate/), with inactivation of any of these genes causing the genetic cancer predisposition syndrome termed Fanconi anemia. ${ }^{9}$ Key components of the FA pathway are the ubiquitin E2 enzyme, UBE2T (also known as FANCT) and the RING-type ubiquitin E3 ligase, FANCL. ${ }^{10}$ In response to the stalling of replication forks at sites of DNA ICLs, UBE2T functions with FANCL and the multiprotein FA complex to monoubiquitylate both subunits of the heterodimeric FANCD2-FANCI (ID) complex. The monoubiquitylated ID complex is then recruited to and retained at sites of ICL lesions and provides a platform for coordinating DNA repair events. When the repair process is completed, the ID complex is deubiquitylated and dissociated from the repaired ICL site by the USP1-UAF1 complex and released from the DNA. ${ }^{11}$

Ubiquitin conjugation is dependent on many proteinprotein interactions (PPIs), and the efficient formation and

Received: July 18, 2019

Accepted: September 16, 2019

Published: September 16, 2019 
disassociation of protein complexes. Therefore, despite ubiquitin conjugating proteins possessing enzymatic activity, it is perhaps more apt to classify them as PPI targets. In drug and chemical probe discovery, such targets are viewed as challenging. This is perhaps reflected by the scarcity of selective small molecule inhibitors of ubiquitin conjugation pathways reported to date. ${ }^{12}$

To identify small-molecule inhibitors of the FA pathway, we developed a high-throughput screen (HTS) compatible assay based on the FA ubiquitylation cascade (see Figure 1a, as well

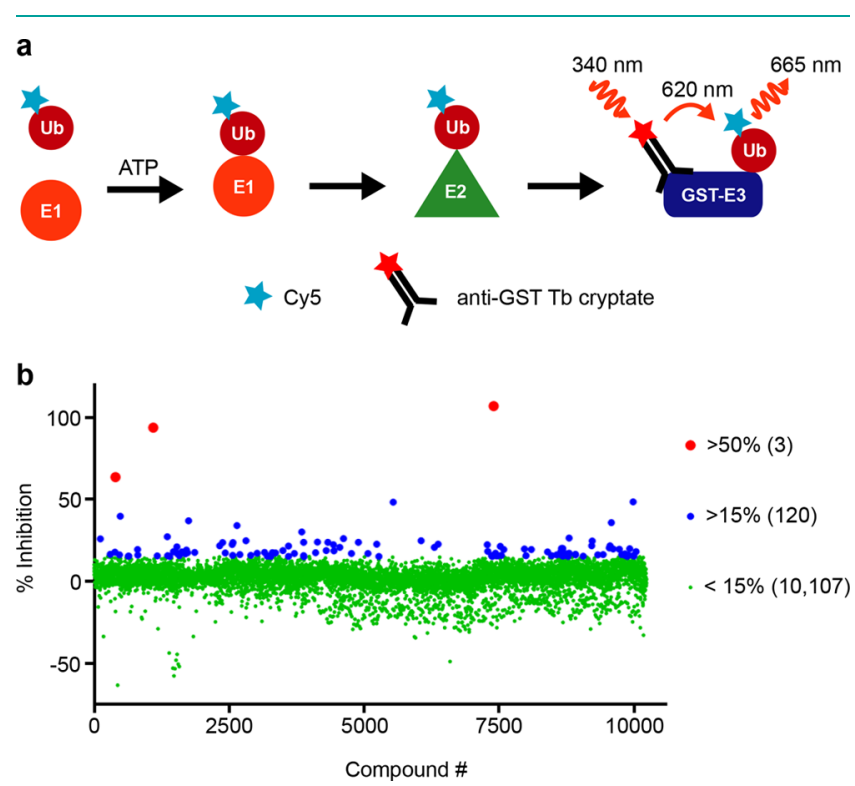

Figure 1. Screening for inhibitors of the FA pathway. (a) Schematic of the HTRF ubiquitylation assay. Ubiquitylation of GST-tagged E3 $\left(\right.$ FANCL $_{\text {RING }}$ ) by the E2 (UBE2T) places Cy5-labeled ubiquitin in close proximity to the anti-GST $\mathrm{Tb}$ cryptate. Excitation of the $\mathrm{Tb}$ cryptate donor results in FRET to the Cy5 acceptor. Simultaneous monitoring of the donor emission $(620 \mathrm{~nm})$ and acceptor emission $(665 \mathrm{~nm}$ ) allows for determination of the 665/620 ratio. (b) HTRF screen results showing average inhibition $(n=2)$ produced by compounds at $20 \mu \mathrm{M}$ (in-house diversity library; 10111 compounds) and $10 \mu \mathrm{M}$ (Selleckchem epigenetic library; 119 compounds). Numbers given in parentheses represent the number of compounds per inhibition threshold.

as Figure $\mathrm{S} 1$ in the Supporting Information). Given the complexity of the full FA ubiquitylation cascade, we constructed a simplified ubiquitylation reaction that would be robust for HTS purposes yet still provide many relevant protein species for small molecules to interact with. The recombinant protein assay developed used homogeneous timeresolved fluorescence (HTRF) and contained Cy5-labeled ubiquitin, the E1 enzyme UBE1, the E2 enzyme UBE2T, and the RING domain (residues 275-375) of the E3 FANCL $\left(F{ } L_{\text {RING }}\right.$ ). FANCL $L_{\text {RING }}$ was used as a surrogate substrate for ubiquitylation in the absence of the FA core and FANCD2/FANCI complexes.

Subsequent screening of a leadlike diversity chemical library consisting of $\sim 10000$ compounds $(n=2)$ (robust Z score of $>0.75$ ) (Figure $S 2$ in the Supporting Information) at a concentration of $20 \mu \mathrm{M}$ led to the identification of 120 primary hits, a hit rate of $1.17 \%$ (Figure $1 \mathrm{~b}$ ). The primary hit significance threshold ( $15 \%$ inhibition) was defined as three standard deviations above the mean inhibition across the entire screen. The compound library screened was specifically curated to avoid compounds with known promiscuous mechanisms of action, such as redox cycling and cysteine reactivity.

Of the primary hits, the 32 most potent compounds-in relation to the observed percentage inhibition at $20 \mu \mathrm{M}$-were selected for a 10-point dose-response study. Of the 32 compounds tested, 12 exhibited an $\mathrm{IC}_{50}$ value of $<100 \mu \mathrm{M}$. Fresh stocks of these compounds were sourced from commercial vendors and $\mathrm{IC}_{50}$ values of $<100 \mu \mathrm{M}$ were obtained for 10 compounds (Figure S3; see the example for CU2 in Figure S4 in the Supporting Information). For most compounds, there was a good correlation between the data from the primary screen and the resupplied compounds (Table $S 1$ in the Supporting Information).

The 10 compounds confirmed by dose-response were then tested in an orthogonal assay to rule out interference with the HTRF assay format. Thus, a modified ubiquitylation reaction was run with biotinylated ubiquitin and in the absence of the anti-GST-Tb antibody. The reaction components were then separated by SDS-polyacrylamide gel electrophoresis and immunoblotted. Notably, no ubiquitylation of FANCL $_{\text {RING }}$ was observed in the presence of compounds CU1, CU2, CU9, CU10, or CU12 at $100 \mu \mathrm{M}$, as indicated by the absence of the band corresponding to monoubiquitylated (mUb)-GSTFANCL $_{\text {RING }}$ (Figure 2a, top panel). However, CU10 and CU12 also prevented the ubiquitylation of UBE2T (Figure S5 in the Supporting Information) and, in addition, somewhat impaired the autoubiquitylation of UBE1 (Figure 2a, bottom panel). Encouragingly, CU1, CU2, and CU9 did not affect ubiquitylation of either UBE2T or UBE1 (see Figure 2a, as well as Figure S5), thereby suggesting some target selectivity.

In order to explore the selectivity of CU1, CU2, CU9, CU10, and CU12, we conducted a counter-screen against other E2 and E3 combinations. Since members of the UBE2D family of E2s are known to ubiquitylate multiple substrates in vitro, ${ }^{13}$ a ubiquitylation assay featuring UBE2D1 and GSTFANCL $_{\text {RING }}$ was performed. With UBE2D1, multiple ubiq-

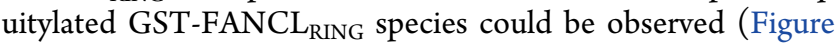
$2 b)$. As with UBE2T and GST-FANCL ${ }_{\text {RING }}$ CU1, CU2, CU9, CU10, and CU12 essentially abolished the ubiquitylation of GST-FANCL $_{\text {RING. }}$ CU10 and CU12 also prevented the formation of any ubiquitylated UBE2D1 species.

A further ubiquitylation assay was performed with an alternative E3, RNF8, which also plays important roles in the $\mathrm{DDR}^{14}$ In contrast to GST-FANCL ${ }_{\mathrm{RING}}$, long polyubiquitin chains were attached to GST-RNF8 by UBE2D1 (Figure 2c). Consistent with our previous observations, CU10 and CU12 appeared to inhibit the ubiquitylation of all species in the assay. In support of our data indicating the selectivity of CU 1, CU2, and CU9, these compounds had no detectable effects on ubiquitylation of GST-RNF8 and UBE2D1.

The lack of an inhibitory effect on the ubiquitylation of UBE1, UBE2T, UBE2D1, and RNF8 by CU1, CU2, and CU9 suggested that these compounds bind to a specific species in the ubiquitylation reactions and are not acting via a pan-assay interference (PAIN)-type mechanism. Furthermore, CU1, CU2, and CU9 appeared to exhibit some degree of selectivity for FANCL-dependent ubiquitylation, although not necessarily selectivity for a particular E2. We did not observe binding of CU2 to UBE2T by differential scanning fluorimetry, ligandobserved NMR, or native mass spectrometry (unpublished data). Taken together, these findings suggest that the target for CU1, CU2, and CU9 is potentially the RING domain of 
a
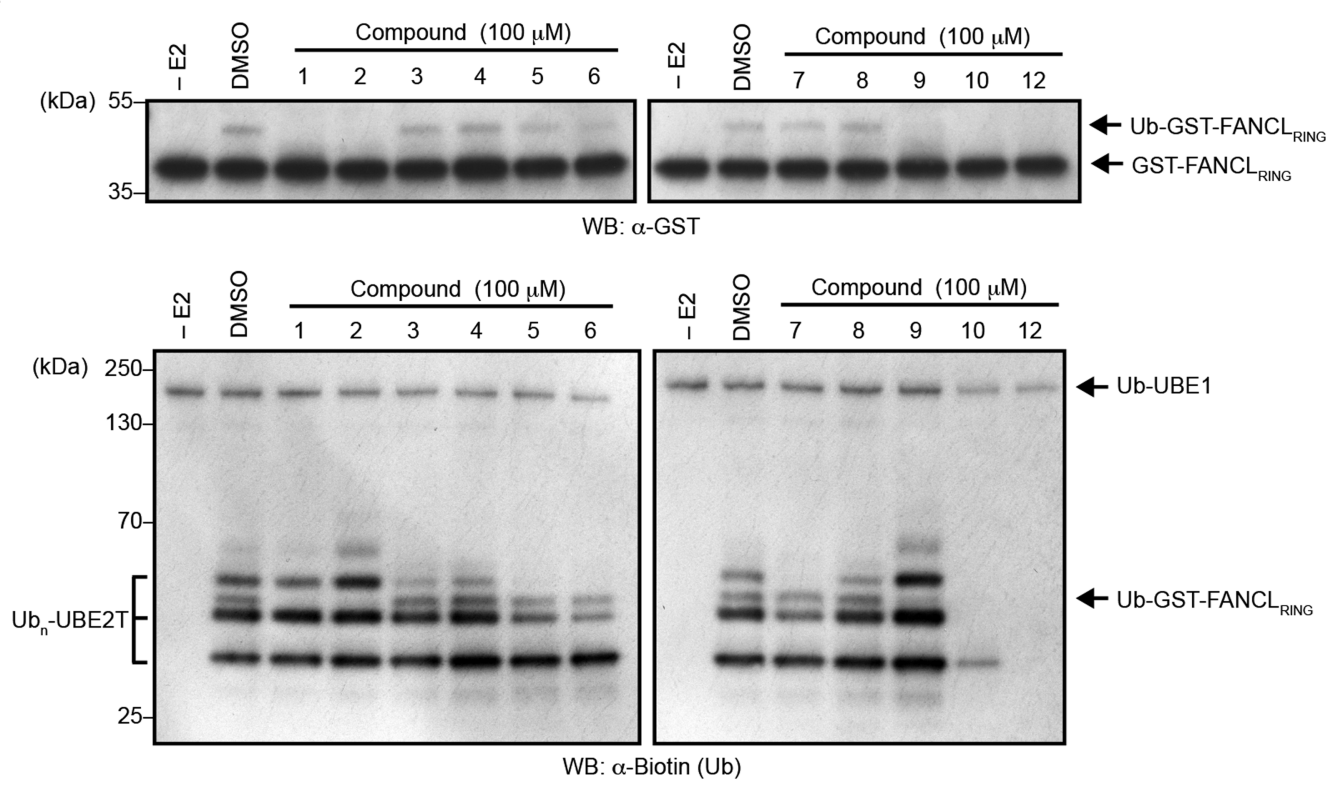

b

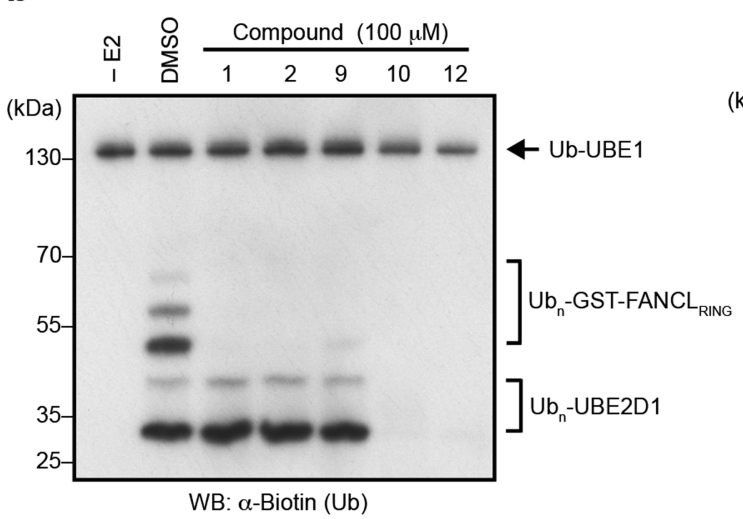

C

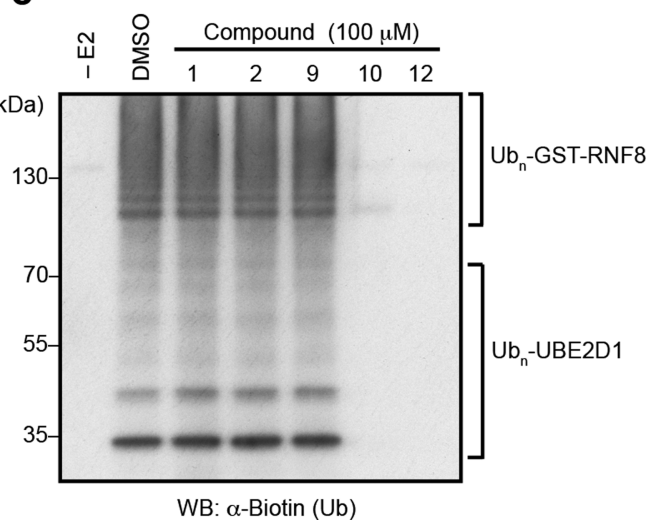

Figure 2. Gel-based ubiquitylation assays with confirmed HTRF hits. CU1, CU2, and CU9 inhibit the ubiquitylation of GST-FANCL RING $_{\text {, but not }}$ GST-RNF8. Western blots (WB) probed for GST or biotin as labeled. Reactions contained 20 nM His 6 -UBE1, 50 nM E2, 250 nM GST-E3, 500 nM biotinylated ubiquitin: (a) E2 = UBE2T, E3 = GST-FANCL RING, 30 min of reaction at $37^{\circ} \mathrm{C}$; (b) E2 = UBE2D1, E3 = GST-FANCL ${ }_{\mathrm{RING}}, 30$ min of reaction at $37{ }^{\circ} \mathrm{C}$; and (c) E2 = UBE2D1, E3 = GST-RNF8, 10 min of reaction at $37^{\circ} \mathrm{C}$.

FANCL or a protein complex comprised thereof; however, further experimental evidence will be required to confirm this.

Next, CU1, CU2, and CU9 were evaluated for their ability to inhibit FANCL-dependent ubiquitylation events in cells. CU10 and CU12 were deprioritized for cellular evaluation due to their lack of selectivity in the in vitro gel-based ubiquitylation assays. The only known in vivo function of UBE2T and FANCL is their direct role in the monoubiquitylation of the heterodimeric FANCD2-FANCI complex, which occurs in response to certain types of DNA damage. Hydroxyurea (HU) is a potent inhibitor of the enzyme ribonucleotide reductase (RNR) and is an antineoplastic drug used in the clinic. ${ }^{15}$ Inhibition of RNR limits de novo dNTP synthesis, causing depletion of cellular dNTP pools, which, in turn, leads to stalled and collapsed replication forks and cell cycle arrest in S-phase. Because of the role of the FA pathway in the stabilization and repair of stalled and collapsed replication forks, $\mathrm{HU}$ is a strong inducer of the UBE2T and FANCL-dependent monoubiquitylation of FANCD2 (mUbFANCD2). ${ }^{16}$
U2OS cells were treated with $\mathrm{HU}$ and the formation of mUb-FANCD2 in the presence of CU 1, CU2, and CU9 was evaluated relative to a DMSO control. Following a 1-h preincubation with the compounds, cells were treated with 2.5 $\mathrm{mM}$ of $\mathrm{HU}$ for $24 \mathrm{~h}$. Biochemically, at $100 \mu \mathrm{M}, \mathrm{CU}$ 1, CU2, and CU9 completely inhibited the ubiquitylation of GSTFANCL $_{\text {RING }}$. However, consistent with the decrease in potency often observed in cellular assays, relative to in vitro biochemical assays, ${ }^{17}$ when cells were treated with CU 1, CU2, and CU9 at $100 \mu \mathrm{M}$, no significant decrease in the level of mUb-FANCD2 was observed (see Figure S6 in the Supporting Information). Accordingly, cells were treated with a higher concentration of compounds in further experiments. In these studies, we also decreased the assay time from $24 \mathrm{~h}$ to $8 \mathrm{~h}$ to mitigate potential compound instability. Thus, we found that cells tolerated CU2 and CU9 at $500 \mu \mathrm{M}$, while CU1 was found to be cytotoxic above $300 \mu \mathrm{M}$. Notably, both CU1 $(200 \mu \mathrm{M})$ and CU2 (500 $\mu \mathrm{M})$ reduced the level of mUb-FANCD2 in response to $\mathrm{HU}$ treatment (see Figures $3 \mathrm{a}$ and $3 \mathrm{~b})$. CU2 $(500 \mu \mathrm{M})$ also reduced the level of $\mathrm{mUb}-\mathrm{FANCD} 2$ generated in response to cisplatin $(10 \mu \mathrm{M})$ treatment for 6, 12, and $24 \mathrm{~h}$ (Figure 3c). 


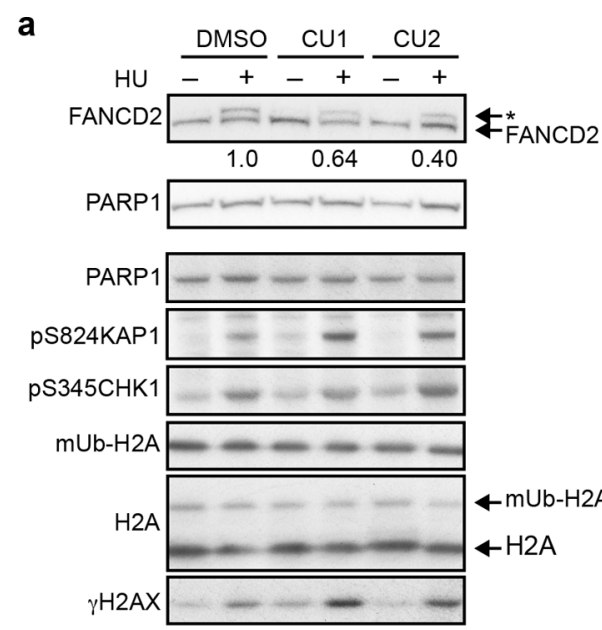

b

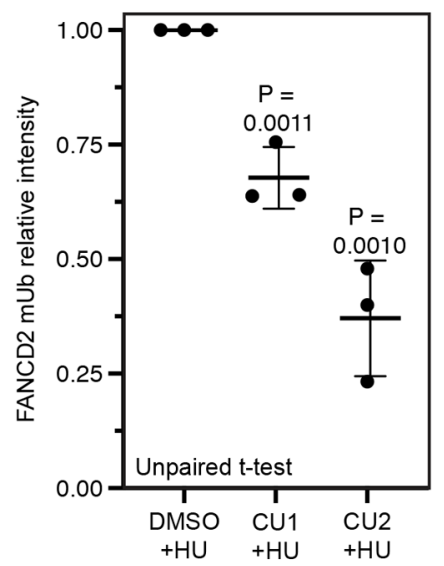

d
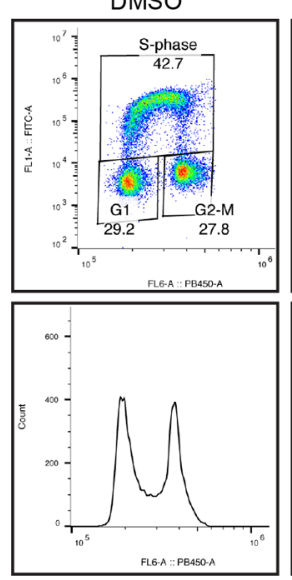

C

Time $\quad 0 \mathrm{hr} \quad 6 \mathrm{hr} \quad 6 \mathrm{hr}$

$\mathrm{CU} 2-+\quad+-+$

cisplatin - $\quad$ - $\quad-\quad-\quad+\quad+$

FANCD2 $\square-\cdots+\cdots$ FANCD2

PARP1 $=-\square-\square$

Time $0 \mathrm{hr} \quad 12 \mathrm{hr} \quad 12 \mathrm{hr}$

CU2 - + - +-+

cisplatin $\quad-\quad-\quad-\quad+\quad+$

FANCD2 $-\square=2$ FANCD2

PARP1 $\square-\square-\square$

Time $0 \mathrm{hr} \quad 24 \mathrm{hr} 24 \mathrm{hr}$

CU2 - +-+-+

cisplatin - $-\quad-\quad+\quad+$

FANCD2 $-\ldots-\square$ FANCD2

PARP1 - - - -

1.00 .53

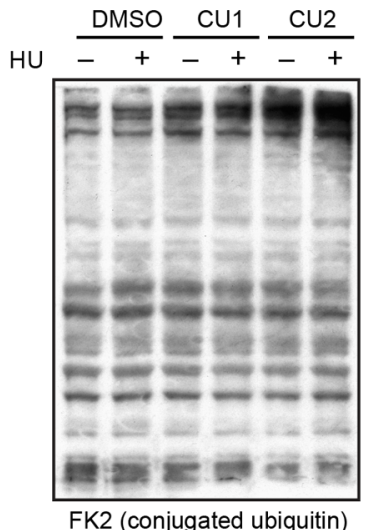

CU1
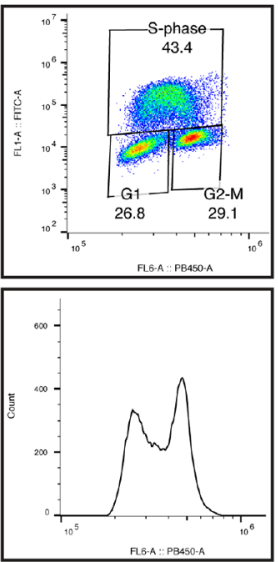
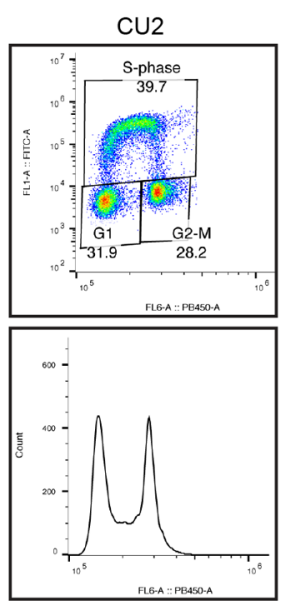

Figure 3. Cell-based assays with CU1 and CU2: (a) Western blot showing that the formation of mUb-FANCD2 $(*)$, in response to HU treatment $(2.5 \mathrm{mM}, 8 \mathrm{~h})$, is impaired in U2OS cells in the presence of CU1 $(200 \mu \mathrm{M})$ or CU2 $(500 \mu \mathrm{M})$. Ratio $=$ mUb-FANCD2/FANCD2 (normalized to DMSO control). mUb-FANCD2 was resolved on a separate gel. PARP1 was used as a loading control for each gel, as well as to assess apoptosis. (b) Quantification of the reduction in mUb-FANCD2, in response to HU treatment $(2.5 \mathrm{mM}, 8 \mathrm{~h})$ in the presence of CU1 (200 $\mu \mathrm{M})$ or CU2 (500 $\mu \mathrm{M}$ ). Mean calculated from three independent experiments. (c) Western blot showing that formation of mUb-FANCD2 (denoted by an asterisk symbol, *) in response to cisplatin treatment $(10 \mu \mathrm{M})$ for 6,12 , and $24 \mathrm{~h}$ is impaired in U2OS cells in the presence of CU2 $(500 \mu \mathrm{M})$. Ratio $=$ mUb-FANCD2/FANCD2 (normalized to DMSO control). (d) Cell cycle analyses based upon EdU incorporation in cells treated with HU (2.5 $\mathrm{mM}, 8 \mathrm{~h})$ in the presence or absence of CU1 $(200 \mu \mathrm{M})$ or CU2 $(500 \mu \mathrm{M})$. HU treatment was used for gating purposes. (e) Western blot showing that CU1 $(200 \mu \mathrm{M})$ or CU2 $(500 \mu \mathrm{M})$ do not affect total protein ubiquitylation with or without HU treatment $(2.5 \mathrm{mM}, 8 \mathrm{~h})$, as assessed by probing with the FK2 antibody.

As DNA damage induced by $\mathrm{HU}$ is cell-cycle-dependent, it was necessary to establish whether CU1 and CU2 might simply prevent cells from entering the S-phase, which would also manifest itself as a reduction in mUb-FANCD2. This was 


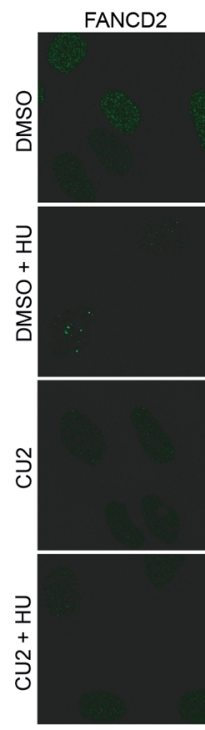

C
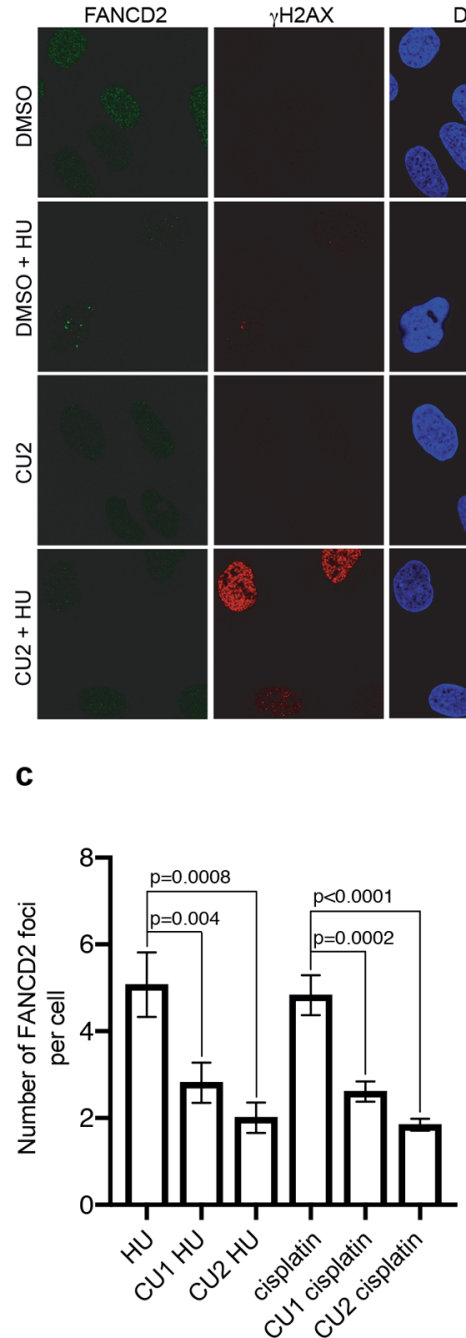

b
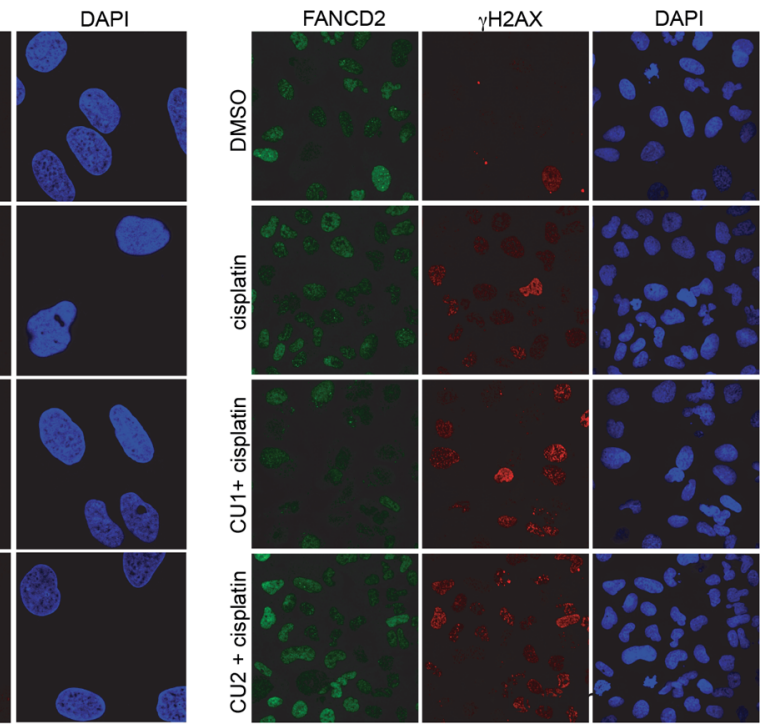

d

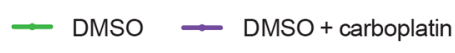

$-\mathrm{CU} 2 \rightarrow \mathrm{CU} 2+$ carboplatin

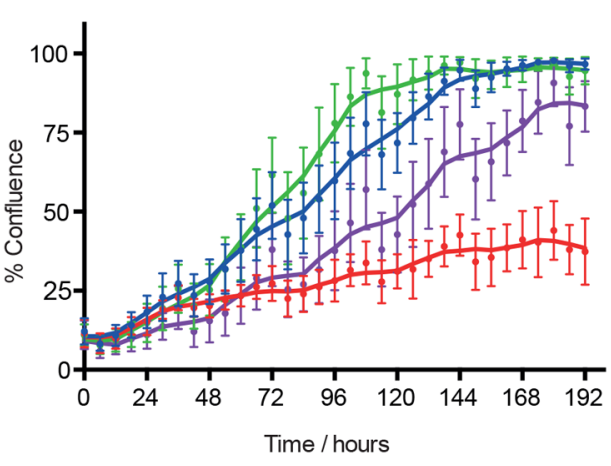

Figure 4. Further cell-based assays with CU1 and CU2: (a, b) Representative immunofluorescence images of U2OS cells treated with HU (2.5 $\mathrm{mM}, 8 \mathrm{~h})$ or cisplatin $(10 \mu \mathrm{M}, 8 \mathrm{~h})$ in the presence or absence of CU1 $(200 \mu \mathrm{M})$ or CU2 $(500 \mu \mathrm{M})$. (c) Quantification of the number of FANCD2 foci in cells treated as in panels (a) and (b). Data accumulated over three independent experiments, each consisting of three technical replicas. (d) IncuCyte analysis of U2OS cells treated with or without carboplatin $(15 \mu \mathrm{M})$ and CU2 $(250 \mu \mathrm{M})$. Mean calculated from three wells, four images per well. $(\mathrm{HU}=\mathrm{DMSO}+\mathrm{HU}$, cisplatin $=\mathrm{DMSO}+$ cisplatin $)$.

determined by analysis of several biomarkers linked to DNA damage in cycling cells. Activated CHK1 (pS345 CHK1) phosphorylates CDC25A, inducing the transient arrest of cells in the S-phase, or in G2 before the onset of mitosis. ${ }^{18}$ Comparable levels of pS345 CHK1 were observed in cells treated with either CU1 or CU2, relative to the DMSO control, indicating that the compounds were not preventing cells from entering the S-phase (Figure 3a). These observations were also confirmed by cell cycle analysis. However, a slightly lower EdU (5-ethynyl-2'-deoxyuridine) incorporation intensity, but not S-phase index, was observed following CU1 treatment, suggesting that it may slow progression through the $S$-phase (Figure $3 \mathrm{~d}$ ).

Following the generation of DNA DSBs, ATM, ATR, and/or DNA-PKcs mediated phosphorylation of histone H2AX generates the phosphorylated species, $\gamma \mathrm{H} 2 \mathrm{AX} .{ }^{19}$ In accord with our conclusion that CU1 and CU2 did not markedly affect progression of cells into and through the S-phase, when used to treat cells in combination with HU, CU1 or CU2 did not decrease and, in fact, somewhat increased the levels of $\gamma \mathrm{H} 2 \mathrm{AX}$, relative to cells treated with $\mathrm{HU}$ plus DMSO control (Figure 3a). Furthermore, these increased levels of $\gamma \mathrm{H} 2 \mathrm{AX}$ suggested that larger numbers of DSBs were generated in HUtreated cells incubated with CU1 or CU2, compared with cells treated with HU and DMSO. Following HU treatment, DSBs are formed if stalled replication forks are not stabilized and subsequently collapse. Therefore, we concluded that CU1 and CU2 appeared to act by impairing the ability of cells to stabilize and repair stalled replication forks. This is consistent with CU1 and CU2 inhibiting the formation of mUbFANCD2, which would otherwise play a role in stabilizing stalled replication forks, thus preventing their collapse and the formation of DSBs.

In response to DSB formation, the transcriptional repressor and RING finger protein, KAP1, is released from chromatin, following its ATM-mediated phosphorylation on Ser824. ${ }^{20}$ In agreement with our findings with $\gamma \mathrm{H} 2 \mathrm{AX}$, increased levels of pS824 KAP1 were observed with both CU1 and CU2, which provides further evidence of the existence of more DSBs/ collapsed replication forks (Figure 3a). An increase in the 
number of DSBs could also have been caused by apoptosis. In cells undergoing apoptosis, PARP1 is cleaved from a full-length $116 \mathrm{kDa}$ protein into 89 and $24 \mathrm{kDa}$ polypeptides by caspase3. ${ }^{21}$ These PARP1 cleavage fragments were not observed when cells were treated with HU plus CU1 or CU2, indicating that the effects we observed on H2AX and KAP1 phosphorylation were not due to apoptosis and, instead, were likely due to the compounds inducing a defect in the FA pathway (Figure 3a).

To investigate if the compounds were affecting more widespread ubiquitylation events in the cell, the monoubiquitylation status of histone $\mathrm{H} 2 \mathrm{~A}$ was assessed (mUb-H2A; Figure $3 a)$. The polycomb repressive complex 1 (PRC1) mediates ubiquitylation of histone $\mathrm{H} 2 \mathrm{~A}$ through its E3 ligase subunits, RING1A and BMI1, in combination with UBE2D3. ${ }^{22}$ In addition, the total level of monoubiquitinylated and polyubiquitinylated conjugates in the cell was assessed by immunoblotting of cell extracts with the FK2 antibody, which recognizes conjugated ubiquitin (Figure $3 \mathrm{e}$ ). mUb$\mathrm{H} 2 \mathrm{~A}$ and total protein ubiquitylation levels were not detectably affected in the presence of CU1 or CU2, suggesting that these compounds are not pan-ubiquitylation inhibitors.

In response to DNA damage, mUb-FANCD2 accumulates at sites of DNA lesions to form foci, promote DNA-damage sensing, activate cell-cycle checkpoints, and promote DNA repair. ${ }^{23}$ Therefore, we employed high-throughput highcontent indirect immunofluorescence studies to examine the effect of CU1 and CU2 on mUb-FANCD2 focus formation. The experimental format used was similar to the immunoblotbased experiments, excepting that, following treatment with $\mathrm{HU}(2.5 \mathrm{mM}$ for $8 \mathrm{~h})$ or cisplatin $(10 \mu \mathrm{M}$ for $8 \mathrm{~h})$, cells were fixed, permeabilized and stained with specific FANCD2 primary and fluorescent secondary antibodies. In addition to monitoring mUb-FANCD2 foci, $\gamma \mathrm{H} 2 \mathrm{AX}$ was evaluated as a marker of DNA damage induction, and cell nuclei were visualized by 4',6-diamidino-2-phenylindole (DAPI) staining. We observed a clear reduction in the number of FANCD2 foci per cell in CU1- or CU2-treated cells in the presence of HU or cisplatin, relative to $\mathrm{HU}$ or cisplatin alone (Figures $4 \mathrm{a}, 4 \mathrm{~b}$, and $4 c)$. These observations are in accord with the immunoblotbased experiments wherein reductions in mUb-FANCD2 were observed (Figure 3a). Although $\gamma \mathrm{H} 2 \mathrm{AX}$ levels appear to be higher in the HU-treated cells (Figure 3a), as was the case in the immunoblot assay, the $p$-value of this increase did not pass the statistical threshold $(p<0.05)$. No such apparent increase was observed in the case of cisplatin treatment (see Figure S7 in the Supporting Information). The quantitative resultsnamely, no reduction in $\gamma \mathrm{H} 2 \mathrm{AX}$ positive cells treated with CU1 or CU2 in combination with $\mathrm{HU}$ or cisplatin-are consistent with the cell cycle analyses indicating that CU1 or CU2 lead to no reduction in S-phase indices.

Finally, to investigate if $\mathbf{C U} 1$ and $\mathbf{C U} 2$ could sensitize cells to a more clinically relevant chemotherapeutic agent, quantitative live cell analyses were performed using the IncuCyte platform. As the FA pathway is involved in the repair of ICLs, cells were treated with the ICL-inducing chemotherapeutic drug, carboplatin. For this experiment, U2OS cells were seeded at a relatively low $(<10 \%)$ density and preincubated with CU1 or CU2 for $1 \mathrm{~h}$. Carboplatin was then added to the cells and images were taken every $6 \mathrm{~h}$ for 8 days in order to monitor growth and proliferation of the cells. Although $500 \mu \mathrm{M}$ CU2 was used in the immunoblot- and immunofluorescence-based experiments, this concentration was found to be cytotoxic/cytostatic upon longer cellular exposures. In contrast, $\mathbf{C U 2}$ at $250 \mu \mathrm{M}$ had a minimal effect on cell growth, with cells reaching $100 \%$ confluency after 6 days, a similar situation to when the cell line was treated with DMSO alone (Figure $4 \mathrm{~d}$ ). The addition of $15 \mu \mathrm{M}$ carboplatin to DMSO-treated cells led to a clear reduction in proliferation rate, and, after 8 days, the cells had still not reached $100 \%$ confluency. The combination of $250 \mu \mathrm{M}$ CU2 with $15 \mu \mathrm{M}$ carboplatin led to an even more pronounced decrease in cell proliferation/growth, with the cells being $<50 \%$ confluent after 8 days (Figure 4d; as shown in Figure S8 in the Supporting Information, while the effect of CU1 was also examined, it was found to strongly inhibit cell growth at concentrations of $>50$ $\mu \mathrm{M}$ and the combined effect of $50 \mu \mathrm{M}$ CU1 with $15 \mu \mathrm{M}$ carboplatin was far less pronounced than that observed for CU2).

In summary, the small-molecule screening campaign and follow-up studies described herein represent a potential starting point for the development of a chemical probe or therapeutic agent targeting the FA pathway. One of the compounds identified, CU2, exhibits promising selectivity in biochemical ubiquitylation assays and also demonstrated activity against the FA pathway in cells. Further work will be necessary to elucidate the precise nature of the protein or protein complex to which CU2 binds. Given the relatively low molecular weight $(357 \mathrm{Da})$ of $\mathbf{C U} 2$, there is certainly scope to improve its cellular potency and target selectivity through chemical synthesis. CU1 represents a less-attractive starting point, in view of the cytotoxicity that we observed. A more potent inhibitor of the FA pathway could find clinical application in sensitizing cancer cells to DNA cross-linking agents (such as carboplatin or mitomycin C). A selective FA pathway inhibitor could also be utilized in the identification and exploitation of synthetic lethal interactions in cancer cells with certain genetic backgrounds. ${ }^{24}$

\section{MATERIALS AND METHODS}

Detailed methods are described in the Supporting Information.

\section{ASSOCIATED CONTENT}

\section{S Supporting Information}

The Supporting Information is available free of charge on the ACS Publications website at DOI: 10.1021/acschembio.9b00570.

Experimental procedures, Figures $\mathrm{S} 1-\mathrm{S} 9$, and Tables S1-S4 (PDF)

\section{AUTHOR INFORMATION}

\section{Corresponding Author}

*E-mail: y.galanty@gurdon.cam.ac.uk.

ORCID

Yaron Galanty: 0000-0001-7167-9004

\section{Present Address}

${ }^{\S}$ Charles River Laboratories, Little Walden, Essex, U.K.

\section{Notes}

The authors declare no competing financial interest.

\section{ACKNOWLEDGMENTS}

We thank Kate Dry and Francisco Muñoz-Martinez for expert editorial assistance and help with the flow cytometry assays, respectively, and the P. Cohen Laboratory (MRC Protein Phosphorylation and Ubiquitylation Unit, University of 
Dundee, UK) for plasmids containing the UBE2T or UBE2D1 cDNA. We thank A. Dutta (University of Virginia, USA) and Y. Machida (Mayo Clinic, Rochester, USA) for the GST-

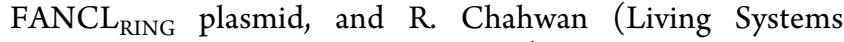
Institute, University of Exeter, U.K.) for the GST-RNF8 plasmid. M.J.C. was funded through the Cambridge $\mathrm{PhD}$ Training Programme in Chemical Biology and Molecular Medicine. Y.G. is funded by Cancer Research UK (No. C6/ A18796) and a Wellcome Trust Investigator Award to S.P.J. (No. 206388/Z/17/Z). Research in the Jackson laboratory is funded by CRUK (No. C6/A18796) and the Wellcome Trust (No. 206388/Z/17/Z), with core infrastructure funding from the Wellcome Trust (No. 203144) and CRUK (No. C6946/ A24843). Work in the CRUK Manchester Institute Drug Discovery Unit was funded by CRUK (Grant Nos. C480/ A1141 and C309/A8274).

\section{REFERENCES}

(1) Hershko, A., and Ciechanover, A. (1998) the Ubiquitin System. Annu. Rev. Biochem. 67, 425-479.

(2) Popovic, D., Vucic, D., and Dikic, I. (2014) Ubiquitination in disease pathogenesis and treatment. Nat. Med. 20, 1242-1253.

(3) Jackson, S. P., and Durocher, D. (2013) Regulation of DNA Damage Responses by Ubiquitin and SUMO. Mol. Cell 49, 795-807.

(4) Jackson, S. P., and Bartek, J. (2009) The DNA-damage response in human biology and disease. Nature 461, 1071-1078.

(5) Ceccaldi, R., Sarangi, P., and D'Andrea, A. D. (2016) The Fanconi anaemia pathway: new players and new functions. Nat. Rev. Mol. Cell Biol. 17I, 337-349.

(6) Galluzzi, L., Senovilla, L., Vitale, I., Michels, J., Martins, I., Kepp, O., Castedo, M., and Kroemer, G. (2012) Molecular mechanisms of cisplatin resistance. Oncogene 31, 1869-1883.

(7) Chirnomas, D., Taniguchi, T., de la Vega, M., Vaidya, A. P., Vasserman, M., Hartman, A.-R., Kennedy, R., Foster, R., Mahoney, J., Seiden, M. V., and D'Andrea, A. D. (2006) Chemosensitization to cisplatin by inhibitors of the Fanconi anemia/BRCA pathway. Mol. Cancer Ther. 5, 952-961.

(8) Voter, A. F., Manthei, K. A., and Keck, J. L. (2016) A HighThroughput Screening Strategy to Identify Protein-Protein Interaction Inhibitors That Block the Fanconi Anemia DNA Repair Pathway. J. Biomol. Screening 21, 626-633.

(9) Michl, J., Zimmer, J., and Tarsounas, M. (2016) Interplay between Fanconi anemia and homologous recombination pathways in genome integrity. EMBO J. 35, 909-923.

(10) Machida, Y. J., Machida, Y., Chen, Y., Gurtan, A. M., Kupfer, G. M., D'Andrea, A. D., and Dutta, A. (2006) UBE2T is the E2 in the Fanconi anemia pathway and undergoes negative autoregulation. Mol. Cell 23, 589-596.

(11) Nijman, S. M. B., Huang, T. T., Dirac, A. M. G., Brummelkamp, T. R., Kerkhoven, R. M., D'Andrea, A. D., and Bernards, R. (2005) The deubiquitinating enzyme USP1 regulates the fanconi anemia pathway. Mol. Cell 17, 331-339.

(12) Huang, X., and Dixit, V. M. (2016) Drugging the undruggables: exploring the ubiquitin system for drug development. Cell Res. 26, 484-498.

(13) Brzovic, P. S., and Klevit, R. E. (2006) Ubiquitin transfer from the E2 perspective: Why is UbcH5 so promiscuous? Cell Cycle 5, 2867-2873.

(14) Kolas, N. K., Chapman, J. R., Nakada, S., Ylanko, J., Chahwan, R., Sweeney, F. D., Panier, S., Mendez, M., Wildenhain, J., Thomson, T. M., Pelletier, L., Jackson, S. P., and Durocher, D. (2007) Orchestration of the DNA-damage response by the RNF8 ubiquitin ligase. Science 318, 1637-1640.

(15) Madaan, K., Kaushik, D., and Verma, T. (2012) Hydroxyurea: a key player in cancer chemotherapy. Expert Rev. Anticancer Ther. 12, 19-29.
(16) Howlett, N. G., Taniguchi, T., Durkin, S. G., D’Andrea, A. D. and Glover, T. W. (2005) The Fanconi anemia pathway is required for the DNA replication stress respone and for the regulation of common fragile site stability. Hum. Mol. Genet. 14, 693-701.

(17) Teuscher, K. B., Zhang, M., and Ji, H. (2017) A Versatile Method to Determine the Cellular Bioavailability of Small-Molecule Inhibitors. J. Med. Chem. 60, 157-169.

(18) Donzelli, M., and Draetta, G. F. (2003) Regulating mammalian checkpoints through Cdc25 inactivation. EMBO Rep. 4, 671-677.

(19) Kuo, L. J., and Yang, L.-X. (2008) Gamma-H2AX-A novel biomarker for DNA double-strand breaks. In Vivo 22, 305-309.

(20) Goodarzi, A. A., Noon, A. T., Deckbar, D., Ziv, Y., Shiloh, Y., Löbrich, M., and Jeggo, P. A. (2008) ATM Signaling Facilitates Repair of DNA Double-Strand Breaks Associated with Heterochromatin. Mol. Cell 31, 167-177.

(21) Boulares, A. H., Yakovlev, A. G., Ivanova, V., Stoica, B. A., Wang, G., Iyer, S., and Smulson, M. (1999) Role of poly(ADP-ribose) polymerase (PARP) cleavage in apoptosis. Caspase 3-resistant PARP mutant increases rates of apoptosis in transfected cells. J. Biol. Chem. 274, 22932-22940.

(22) McGinty, R. K., Henrici, R. C., and Tan, S. (2014) Crystal structure of the PRC1 ubiquitylation module bound to the nucleosome. Nature 514, 591-596.

(23) Garcia-Higuera, I., Taniguchi, T., Ganesan, S., Meyn, M. S., Timmers, C., Hejna, J., Grompe, M., and D'Andrea, A. D. (2001) Interaction of the Fanconi anemia proteins and BRCA1 in a common pathway. Mol. Cell 7, 249-262.

(24) Jenkins, C., Kan, J., and Hoatlin, M. E. (2012) Targeting the fanconi anemia pathway to identify tailored anticancer therapeutics. Anemia 2012, 481583. 